\title{
Fast and accurate algorithm for the computation of complex linear canonical transforms
}

\author{
Aykut Koç, ${ }^{1, *}$ Haldun M. Ozaktas, ${ }^{2}$ and Lambertus Hesselink ${ }^{1}$ \\ ${ }^{1}$ Department of Electrical Engineering, Stanford University, Stanford, California 94305, USA \\ ${ }^{2}$ Department of Electrical Engineering, Bilkent University, TR-06800 Bilkent, Ankara, Turkey \\ *Corresponding author: aykutkoc@stanford.edu
}

Received April 1, 2010; revised June 2, 2010; accepted June 30, 2010;

posted July 6, 2010 (Doc. ID 126363); published August 5, 2010

\begin{abstract}
A fast and accurate algorithm is developed for the numerical computation of the family of complex linear canonical transforms (CLCTs), which represent the input-output relationship of complex quadratic-phase systems. Allowing the linear canonical transform parameters to be complex numbers makes it possible to represent paraxial optical systems that involve complex parameters. These include lossy systems such as Gaussian apertures, Gaussian ducts, or complex graded-index media, as well as lossless thin lenses and sections of free space and any arbitrary combinations of them. Complex-ordered fractional Fourier transforms (CFRTs) are a special case of CLCTs, and therefore a fast and accurate algorithm to compute CFRTs is included as a special case of the presented algorithm. The algorithm is based on decomposition of an arbitrary CLCT matrix into real and complex chirp multiplications and Fourier transforms. The samples of the output are obtained from the samples of the input in $\sim N \log N$ time, where $N$ is the number of input samples. A space-bandwidth product tracking formalism is developed to ensure that the number of samples is information-theoretically sufficient to reconstruct the continuous transform, but not unnecessarily redundant. (C) 2010 Optical Society of America

OCIS codes: $070.2580,350.6980,070.2590$.
\end{abstract}

\section{INTRODUCTION}

Linear canonical transforms (LCTs) appear widely in optics [1-3], electromagnetics, and classical and quantum mechanics [4-6], as well as in computational and applied mathematics [7]. The application areas of LCTs include, among others, the study of scattering from periodic potentials [8-10], laser cavities [2,11,12], and multilayered structures in optics and electromagnetics [13].

LCTs with real parameters have received considerably more attention than LCTs with complex parameters [4]. Real linear canonical transforms (RLCTs) are unitary mappings between the elements of Hilbert space of square integrable functions of a variable in R. RLCTs are represented by $2 \times 2$ unimodular real matrices,

$$
\mathbf{M}_{R}=\left[\begin{array}{ll}
a & b \\
c & d
\end{array}\right]
$$

with determinant equal to 1 , where $a, b, c$, and $d$ are real. The parameter matrices $\mathbf{M}_{R}$ form the real symplectic group $S p(2, \mathrm{R})$ with three independent parameters [14]. RLCTs are of great importance in electromagnetic, acoustic, and other wave propagation problems since they represent the solution of the wave equation under a variety of circumstances. At optical frequencies, RLCTs can model a broad class of lossless optical systems including thin lenses, sections of free space in the Fresnel approximation, sections of quadratic graded-index media, and arbitrary concatenations of any number of these, sometimes referred to as first-order optical systems or quadraticphase systems $[1,15-26]$.
The extension of RLCTs to complex linear canonical transforms (CLCTs) is rather involved [4,27-29]. The extension is very briefly summarized as follows. When we let the entries of the unimodular transform matrices be complex numbers, we obtain the unit-determinant matrices,

$$
\mathbf{M}_{C}=\left[\begin{array}{ll}
a & b \\
c & d
\end{array}\right]
$$

where $a, b, c$, and $d$ are complex parameters. The matrices $\mathbf{M}_{C}$ form the complex symplectic group $S p(2$, C) with six independent parameters [29]. However, CLCTs represented by this symplectic group can no longer be established as a unitary mapping between the Hilbert space of square integrable functions in R. Instead, we have a mapping from the Hilbert space of square integrable functions of a real variable to analytical functions of a complex variable on the complex plane in the Bargmann-Hilbert space of square integrable functions [30,31], as established in [4,27-29]. The CLCTs are required to be bounded but not necessarily unitary, in which case we need to represent CLCTs with a semigroup $H S p(2, \mathrm{C})$ within the group $S p(2$, C). More on the mathematical foundations and theory of CLCTs can be found in [4,27-29].

Bilateral Laplace transforms, Bargmann transforms, Gauss-Weierstrass transforms [4,27,32], fractional Laplace transforms [33], and complex-ordered fractional Fourier transforms (CFRTs) [34-37] are all special cases of CLCTs. An important special case of CLCTs is the family of CFRTs. The CFRT is the generalization of the fractional Fourier transform (FRT) where the order of the 
transformation is allowed to be a complex number, and consequently the abcd matrix elements are in general complex. The optical interpretation of the CFRT, its properties, and optical realizations can be found in [34-38]. An interesting property of CFRTs is that, in some restricted cases, they can be optically realized by RLCTs [39].

To avoid confusion, we note that a number of publications have used the term complex fractional Fourier transformation (FT) to refer to a particular generalization of the FRT [40,41], which is not a complex FRT in the sense of the order parameter being a complex number. The entity referred to as a complex FRT in these publications is distinct from what we refer to as a complex FRT and is actually a special case of real two-dimensional (2D) non-separable or non-symmetrical FRTs. Since such transforms are a special case of $2 \mathrm{D}$ non-separable LCTs, their digital computation is covered by the algorithm proposed in [42]. To avoid confusion with this important but distinct entity, we will use the term complex-ordered to refer to complex FRTs belonging to the class of CLCTs. By developing a general algorithm for CLCTs, we also obtain an algorithm for the important special case of CFRTs.

Given its ubiquitous nature and numerous applications, the fast and efficient digital computation of LCTs is of considerable interest. Many works have addressed the problem of sampling and computation of RLCTs, using both decomposition-based and discrete-LCT-based methods [43-51].

The literature and developments on algorithms for real one-dimensional (1D) and real symmetrical (separable) 2D LCTs are reviewed and summarized in [52]. Recent work has also addressed the computation of the more difficult non-symmetrical (non-separable and nonorthogonal) 2D LCTs, which include anamorphic/ astigmatic cases in which the system does not exhibit symmetry about the optical axis [42]. Thus, an appropriate fast algorithm exists for all possible $1 \mathrm{D}$ and $2 \mathrm{D}$ RLCTs. The purpose of this paper is to cover the case of CLCTs. To our knowledge, there is no algorithm in the literature that efficiently calculates CLCTs or even its most prominent special case, the CFRT.

The distinguishing feature of our approach is the way our algorithm carefully addresses sampling and spacebandwidth product issues from an information-theoretical perspective. Special care is taken to ensure that the output samples represent the continuous transform in the Nyquist-Shannon sense during every stage of the algorithm so that the continuous transform can be fully recovered from the samples. Despite the highly oscillatory nature of the integral kernel, we carefully manage the sampling rate so as to ensure that the number of samples used is sufficient, but not much larger than the spacebandwidth product of the input signal so that the algorithms are as efficient as possible. The straightforward method of sampling the input field and the kernel, and then calculating the output field, is not suitable for several reasons. First of all, due to the highly oscillatory nature of the integral kernel, a naive application of the $\mathrm{Ny}$ quist sampling theorem to determine the sampling rate would result in an excessively large number of samples and inefficient computation. On the other hand, ignoring the oscillations of the kernel and determining the sam- pling rate according to the input field alone may cause under-representation of the output field in the NyquistShannon sense. This unacceptable situation arises due to the fact that the particular 2D LCT that we are calculating may increase the space-bandwidth product in one or both of the dimensions. If we do not increase the number of samples that we are working with, so as to compensate for this increase, there will be information loss and we will not be able to recover the true transformed output from our computed samples. The computation of CLCTs involves a number of issues which do not arise in the case of RLCTs. The decompositions employ complex chirp multiplications (CCMs) whose effect on the Wigner distribution (WD) must be clarified to ensure proper spacebandwidth tracking and control.

Complex-parametered LCTs allow several kinds of optical systems to be represented, including lossy as well as lossless ones. When complex parameters are involved, LCTs may no longer be unitary and boundedness issues may arise. The decomposition of general CLCTs into Fourier transforms and real and imaginary CMs allows us to derive conditions on the transform parameters that ensure boundedness.

We also need to find the conditions under which $H S p(2, \mathrm{C})$ can be constructed as a mapping from $\mathbb{R} \rightarrow \mathbb{R}$. This is because we are interested in optical applications of CLCTs where the inputs and outputs are functions of real spatial variables. Such CLCTs which map functions over Hilbert spaces from the real line to the real line are called passive CLCTs in [27], whereas CLCTs that map functions from the real line to analytical functions on complex Bargmann-Hilbert spaces are called active CLCTs. Thus the eligible parameters also depend on how the $H S p(2, \mathrm{C})$ semigroup is constructed for $\mathbb{R} \rightarrow \mathbb{R}$. Wolf derived the parameter spaces for which the CLCTs represent a mapping from $\mathbb{R} \rightarrow \mathbb{R}$ and the output is bounded [27]. However, this construction excludes some optically important special cases like Gaussian apertures. The decompositions we use allow the derivation of conditions which do not exclude Gaussian apertures. The specification of such conditions was not necessary in the RLCT case.

The paper is organized as follows. In Section 2, we give the fundamental definitions and properties of both RLCTs and CLCTs. Section 3 presents some mathematical preliminaries that we use in the derivation of our algorithm and review some special and important CLCTs in optics. In Section 4, we present a careful analysis of every possible case the complex transform matrix may assume and present fast algorithms based on decompositions into generalized CMs, real scalings, and FTs. We also determine whether a given CLCT represents an optically possible bounded input-output relationship from the real line to the real line. Numerical examples to demonstrate the accuracy of the algorithm are given in Section 5. Finally we conclude in Section 6.

\section{LINEAR CANONICAL TRANSFORMS}

We first recall the definition of RLCTs and discuss the group theoretical structure of RLCTs. Then we will give the definition of CLCTs and explain its group theoretical structure. 
The RLCT of $f(u)$ with real parameter matrix $\mathbf{M}_{R}$ is denoted as $f_{\mathbf{M}_{R}}(u)=\left(\mathcal{C}_{\mathbf{M}_{R}} f\right)(u)$,

$$
\begin{gathered}
\left(\mathcal{C}_{\mathbf{M}_{R}} f\right)(u)=\int_{-\infty}^{\infty} K_{R}\left(u, u^{\prime}\right) f\left(u^{\prime}\right) \mathrm{d} u^{\prime}, \\
K_{R}\left(u, u^{\prime}\right)=e^{-i \pi / 4} \sqrt{\beta} \exp \left[i \pi\left(\alpha u^{2}-2 \beta u u^{\prime}+\gamma u^{\prime 2}\right)\right],
\end{gathered}
$$

where $\alpha, \beta$, and $\gamma$ are real parameters independent of $u$ and $u^{\prime}$ and where $\mathcal{C}_{\mathbf{M}_{R}}$ is the RLCT operator. The transform is unitary. The $2 \times 2$ matrix $\mathbf{M}$ whose elements are $a, b, c, d$ represents the same information as the three parameters $\alpha, \beta$, and $\gamma$ which uniquely define the LCT,

$$
\mathbf{M}_{R}=\left[\begin{array}{ll}
a & b \\
c & d
\end{array}\right]=\left[\begin{array}{cc}
\gamma / \beta & 1 / \beta \\
-\beta+\alpha \gamma / \beta & \alpha / \beta
\end{array}\right]=\left[\begin{array}{cc}
\alpha / \beta & -1 / \beta \\
\beta-\alpha \gamma / \beta & \gamma / \beta
\end{array}\right]^{-1} .
$$

The unit-determinant matrix $\mathbf{M}_{R}$ belongs to the class of unimodular matrices. From a group theoretical point of view RLCTs form the three-parameter symplectic group $S p(2, \mathbb{R})$.

The CLCT of $f(u)$ with a complex parameter matrix $\mathbf{M}_{C}$ is denoted as $f_{\mathbf{M}_{C}}(u)=\left(\mathcal{C}_{\mathbf{M}_{C}} f\right)(u)$,

$$
\begin{gathered}
\left(\mathcal{C}_{\mathbf{M}_{C}} f\right)(u)=\int_{-\infty}^{\infty} K_{C}\left(u, u^{\prime}\right) f\left(u^{\prime}\right) \mathrm{d} u^{\prime}, \\
K_{C}\left(u, u^{\prime}\right)=e^{-i \pi / 4} \sqrt{\bar{\beta}} \exp \left[i \pi\left(\bar{\alpha} u^{2}-2 \bar{\beta} u u^{\prime}+\bar{\gamma} u^{\prime 2}\right)\right],
\end{gathered}
$$

where $\bar{\alpha}, \bar{\beta}$, and $\bar{\gamma}$ are complex parameters independent of $u$ and $u^{\prime}$ and where $\mathcal{C}_{\mathbf{M}_{C}}$ is the CLCT operator. $\mathbf{M}_{C}$ again has a unit determinant and is given by

$$
\mathbf{M}_{C}=\left[\begin{array}{ll}
a & b \\
c & d
\end{array}\right]=\left[\begin{array}{ll}
a_{r}+i a_{c} & b_{r}+i b_{c} \\
c_{r}+i c_{c} & d_{r}+i d_{c}
\end{array}\right]=\left[\begin{array}{cc}
\bar{\gamma} / \bar{\beta} & 1 / \bar{\beta} \\
-\bar{\beta}+\bar{\alpha} \bar{\gamma} / \bar{\beta} & \bar{\alpha} / \bar{\beta}
\end{array}\right],
$$

where $a_{r}, a_{c}, b_{r}, b_{c}, c_{r}, c_{c}, d_{r}$, and $d_{c}$ are real numbers. The overbar in the parameters $\bar{\alpha}, \bar{\beta}$, and $\bar{\gamma}$ is to emphasize that these parameters are now complex, corresponding to a total of six real parameters: $\bar{\alpha}=\alpha_{r}+i \alpha_{c}, \bar{\beta}=\beta_{r}+i \beta_{c}$, and $\bar{\gamma}$ $=\gamma_{r}+i \gamma_{c}$. In terms of these parameters the kernel $K_{C}$ can be rewritten as

$$
\begin{aligned}
& K_{C}\left(u, u^{\prime}\right) \\
& \quad=e^{-i \pi / 4} \sqrt{\beta_{r}+i \beta_{c}} e^{i \pi\left(\alpha_{r} u^{2}-2 \beta_{r} u u^{\prime}+\gamma_{r} u^{\prime 2}\right)} e^{-\pi\left(\alpha_{c} u^{2}-2 \beta_{c} u u^{\prime}+\gamma_{c} u^{\prime 2}\right)} .
\end{aligned}
$$

The bidirectional relationship between the $\bar{\alpha}, \bar{\beta}, \bar{\gamma}$ parameters and the matrix entries are given as follows:

$$
\begin{gathered}
\alpha_{r}=\frac{d_{r} b_{r}+d_{c} b_{c}}{b_{r}^{2}+b_{c}^{2}}, \quad \alpha_{c}=\frac{d_{c} b_{r}-d_{r} b_{c}}{b_{r}^{2}+b_{c}^{2}}, \\
\beta_{r}=\frac{b_{r}}{b_{r}^{2}+b_{c}^{2}}, \quad \beta_{c}=\frac{-b_{c}}{b_{r}^{2}+b_{c}^{2}},
\end{gathered}
$$

$$
\begin{gathered}
\gamma_{r}=\frac{a_{r} b_{r}+a_{c} b_{c}}{b_{r}^{2}+b_{c}^{2}}, \quad \gamma_{c}=\frac{a_{c} b_{r}-a_{r} b_{c}}{b_{r}^{2}+b_{c}^{2}}, \\
a_{r}=\frac{\beta_{c} \gamma_{c}+\beta_{r} \gamma_{r}}{\beta_{r}^{2}+\beta_{c}^{2}}, \quad a_{c}=\frac{\beta_{r} \gamma_{c}-\beta_{c} \gamma_{r}}{\beta_{r}^{2}+\beta_{c}^{2}},
\end{gathered}
$$$$
b_{r}=\frac{\beta_{r}}{\beta_{r}^{2}+\beta_{c}^{2}}, \quad b_{c}=\frac{-\beta_{c}}{\beta_{r}^{2}+\beta_{c}^{2}},
$$$$
d_{r}=\frac{\beta_{c} \alpha_{c}+\beta_{r} \alpha_{r}}{\beta_{r}^{2}+\beta_{c}^{2}}, \quad d_{c}=\frac{\alpha_{c} \beta_{r}-\alpha_{r} \beta_{c}}{\beta_{r}^{2}+\beta_{c}^{2}} .
$$

\section{PRELIMINARIES}

\section{A. Wigner Distributions}

Here we will review the relationship between LCTs and the WD, which will aid us in understanding the effects of the elementary blocks used in our decompositions. The $\mathrm{WD}, W_{f}(u, \mu)$, of a signal $f(u)$ can be defined as follows $[53,54]$ :

$$
W_{f}(u, \mu)=\int_{-\infty}^{\infty} f\left(u+u^{\prime} / 2\right) f^{*}\left(u-u^{\prime} / 2\right) e^{-2 \pi i \mu u^{\prime}} \mathrm{d} u^{\prime} .
$$

Roughly speaking, $W(u, \mu)$ is a function which gives the distribution of the signal energy over space and frequency. Its integral over space and frequency, $\int_{-\infty}^{\infty} \int_{-\infty}^{\infty} W(u, \mu) \mathrm{d} u \mathrm{~d} \mu$, gives the signal energy.

Let $f$ denote a signal and $f_{\mathbf{M}}$ be its LCT with parameter matrix M. Then, the WD of $f_{\mathbf{M}}$ can be expressed in terms of the WD of $f$ as [1]

$$
W_{f_{\mathbf{M}}}(u, \mu)=W_{f}(d u-b \mu,-c u+a \mu) .
$$

This means that the WD of the transformed signal is a linearly distorted version of the original distribution. The Jacobian of this coordinate transformation is equal to the determinant of the matrix $\mathbf{M}$, which is unity. Therefore this coordinate transformation does not change the support area of the WD. The invariance of the support area means that LCTs do not concentrate or deconcentrate energy. The support area of the WD can also be approximately interpreted as the number of degrees of freedom of the signal. Therefore, the number of samples needed to represent the signal does not change after a RLCT operation.

For the purpose of space-bandwidth tracking as employed in our algorithm, we do not require a full characterization of the effects of CLCTs on the WD. However, we do need to know the effect of multiplying a function with another function on the WD to derive a space-bandwidth product tracking method for CLCTs. This multiplication property is not required in deriving our previous algorithms for RLCTs $[42,47]$, but it will be necessary in our CLCT algorithm. The WD has the following multiplication property [1]: let $h(u)$ and $f(u)$ be two functions and let $W_{h}(u, \mu)$ and $W_{f}(u, \mu)$ be their corresponding WDs. Then $h(u) f(u)$ has the WD given by 


$$
\int W_{h}\left(u, \mu-\mu^{\prime}\right) W_{f}\left(u, \mu^{\prime}\right) \mathrm{d} \mu^{\prime}
$$

In other words, when two functions are multiplied, the WD of the resulting function is given by the convolution of the WDs of the initial two functions along the frequency dimension.

It is well known that a nonzero function and its FT cannot both be confined to finite intervals. However, in practice we always work with samples of finite extent signals. We assume that a large percentage of the signal energy, as represented by the WD, is confined to an ellipse with diameters $\Delta S$ in the space dimension and $\Delta B$ in the spatial-frequency dimension, which can be ensured by choosing $\Delta S$ and $\Delta B$ suitably. This implies that the spacedomain representation is approximately confined to the interval $[-\Delta S / 2, \Delta S / 2]$ and that the frequency-domain representation is approximately confined to $[-\Delta B / 2$, $\Delta B / 2]$. We then define the space-bandwidth product $\Delta S \Delta B$, which is always $\geq 1$ because of the uncertainty relation. Let us now introduce the scaling parameter $s$ and scaled coordinates, such that the space- and frequencydomain representations are confined to intervals of lengths $\Delta S / s$ and $\Delta B s$. Let $s=\sqrt{\Delta S / \Delta B}$ so that the lengths of both intervals become equal to the dimensionless quantity $\sqrt{\Delta B \Delta S}$, which we denote by $\Delta u$, and the ellipse becomes a circle with diameter $\Delta u$. It will not make much difference if we assume that the signal is contained within the smallest square containing this circle. In the new scaled coordinates, signals can be represented in both domains with $\Delta u^{2}$ samples spaced $\Delta u^{-1}$ apart. We will assume that this dimensional normalization has been performed and that the coordinates $u$ and $\mu$ are dimensionless.

For a signal with rectangular space-frequency support, the space-bandwidth product is equal to the number of degrees of freedom. This is not true for signals with other support shapes [55]. While we have observed that LCTs do not change the number of degrees of freedom of a signal, they may change its space-bandwidth product. This will be evident when we examine some of the basic special cases of the LCT.

\section{B. CLCTs in Optics and Special CLCTs}

Magnification (scaling), FT, real fractional Fourier transformation (RFRT), real chirp multiplication (CM), CCM, Gauss-Weierstrass transform, and CFRT are all special cases of CLCTs that have optical realizations. Scaling, FT, RFRT, and CM, which have real parameters, belong to the narrower class of RLCTs and have been reviewed in [47]. In this section, we only review complex-parametered cases that are essential for our development.

\section{Complex Scaling (Magnification)}

Simple scaling with a real parameter $M$ is an operation which corresponds to optical magnification [47]. If the parameter $M$ is allowed to be complex, we obtain the complex scaling operation, which is a special case of CLCTs. With complex scaling, the real axis on which the input function is defined is mapped to a straight line in the complex plane passing through the origin and making an angle $\arg (M)$ with the real axis. The mapping becomes $\mathbb{R} \rightarrow \mathrm{C}$. The interpretation of complex scaling in quantum mechanics has been discussed in [56,57], while an interpretation from a signal processing perspective can be found in [58]. However, we are not aware of the realization and application of complex scaling from a purely optical point of view.

\section{Gaussian Apertures (Complex Chirp Multiplication)} Gaussian apertures, also called soft apertures, are a special case of CLCTs. They are actually CMs with a complex parameter and are the complex counterparts of CM operations. We will hereafter refer to them as CCM. The definition of CCM is similar to the definition of real CM, where we replace the chirp parameter with a purely imaginary complex parameter,

$$
\begin{aligned}
& \mathcal{C}_{\mathbf{Q}_{i q}} f(u)=\mathcal{Q}_{i q} f(u)=e^{\pi q u^{2}} f(u), \\
& \mathbf{Q}_{i q}=\left[\begin{array}{cc}
1 & 0 \\
-i q & 1
\end{array}\right]=\left[\begin{array}{cc}
1 & 0 \\
i q & 1
\end{array}\right]^{-1},
\end{aligned}
$$

where $q$ is real and so $i q$ is a purely imaginary parameter. It essentially behaves like a multiplicative filter where the transmission is dependent on the transverse dimension quadratic-exponentially. To exclude the unbounded case we require $q \leq 0$.

We now discuss the effect of CCM on the WD. We need this result in order to track and control the spacebandwidth product of CLCTs. This result is not needed in algorithms for RLCTs because there are no CCM stages in RLCTs and is of a considerably different nature than the operations employed there. We use the property given in Eq. (12) with $h(u)=e^{\pi q u^{2}}$. The WD of $h(u)$, denoted by $W_{h}(u, \mu)$, can be obtained by directly using the definition of the WD [Eq. (10)],

$$
W_{h}(u, \mu)=\sqrt{\frac{2}{-q}} e^{2 \pi q u^{2}} e^{(2 \pi / q) \mu^{2}}, \quad q<0 .
$$

The WD of the Gaussian function is a 2D Gaussian function in the space-frequency plane. Since $q<0$, this function decays with increasing $u$ and $\mu$. Therefore, we can specify a rectangular region which contains almost all of the energy of the function. We will choose the extent of this rectangle to correspond to plus/minus four standard deviations of the Gaussian in both the space and frequency dimensions, which defines a rectangle with extent

$$
\begin{aligned}
& g_{1}=\sqrt{16 / \pi|q|}, \\
& g_{2}=\sqrt{16|q| / \pi}
\end{aligned}
$$

in the space and frequency dimensions, respectively. When the WD of the input function and the WD of the Gaussian function are convolved along the $\mu$ direction to find the resulting WD of the output function (as illustrated in Fig. 1), the resulting space extent of the support of the output WD will be given by $\min \left(d_{1}, g_{1}\right)$ and the resulting frequency extent will be given by $d_{2}+g_{2}$, where $d_{1}$ and $d_{2}$ are the space and spatial-frequency extent of the input function. 


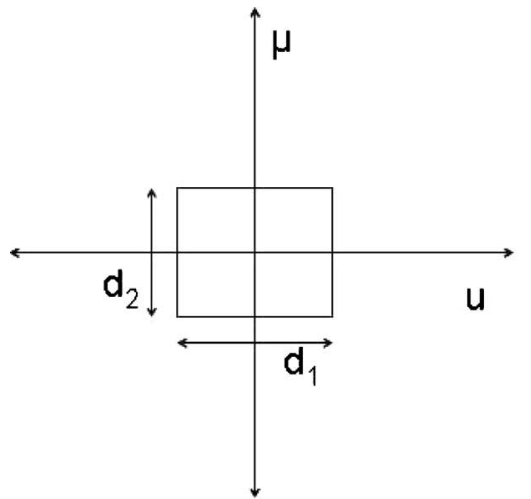

(a)

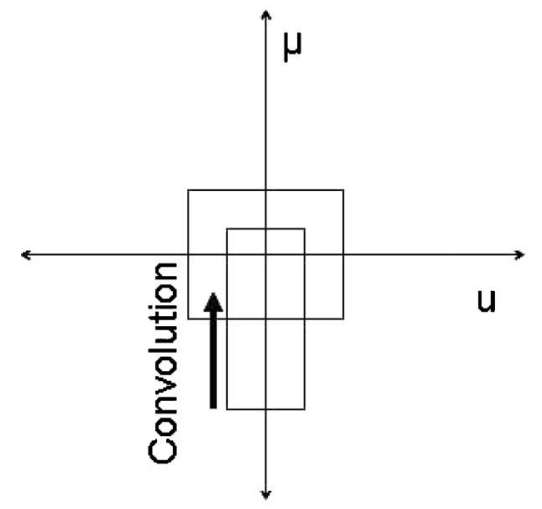

(c)

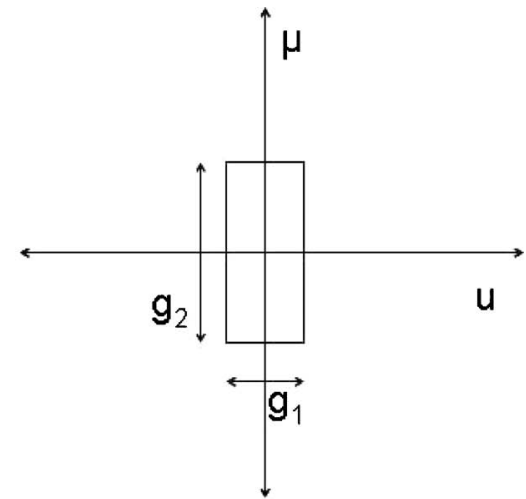

(b)

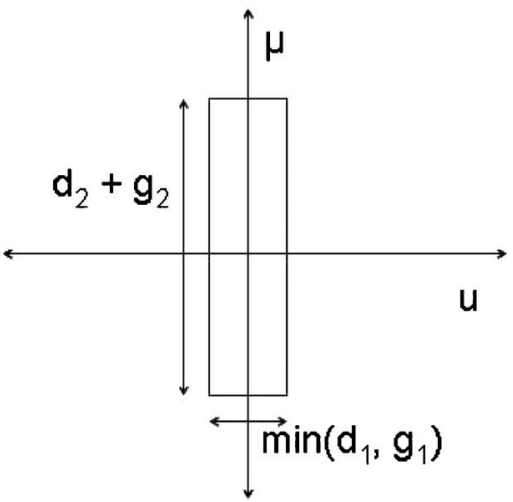

(d)

Fig. 1. The effect of the CCM operation on the WD.

3. Gauss-Weierstrass Transform

The Gauss-Weierstrass transform with parameter $t$ is given by the integral transform [4]

$$
\mathcal{G}_{t} f(u)=\sqrt{1 / t} \int_{-\infty}^{\infty} e^{-\pi\left(u-u^{\prime}\right)^{2} / t} f\left(u^{\prime}\right) \mathrm{d} u^{\prime} .
$$

It gives the solution of the heat equation. The complex chirp convolution (CCC) operation, which is a special case of CLCTs, is represented by the transform matrix

$$
\mathbf{R}_{i r}=\left[\begin{array}{cc}
1 & i r \\
0 & 1
\end{array}\right]
$$

and is equivalent to convolution by a Gaussian function,

$$
\mathcal{C}_{\mathbf{R}_{i r}} f(u)=\mathcal{R}_{i r} f(u)=f(u) * e^{i \pi / 4} \sqrt{1 / r} \exp \left(\pi u^{2} / r\right) .
$$

We observe that CCC is the same as the GaussWeierstrass transform when we choose the CCC parameter $r=-t$. As in the case of the FT, there is again the inconsequential constant phase factor $e^{-i \pi / 4}$ difference between the two definitions. CCC operations are covered by our algorithm since they are a special case of CLCTs. CCC operations are most conveniently calculated by expressing them as a FT followed by a CCM operation followed by an inverse FT.

The combined effect of two CCM (or CCC) operations following each other is again a CCM (or CCC) operation, whose parameter is found by summing the parameters of the two constituent operations. If two CCM operations with real or complex parameters $q_{1}$ and $q_{2}$ follow each other, the equivalent operation is a new CCM operation with parameter $q_{1}+q_{2}$. If two CCC operations with real or complex parameters $r_{1}$ and $r_{2}$ follow each other, the equivalent operation is a new CCC operation with parameter $r_{1}+r_{2}$.

\section{CFRT}

The $a$ th order RFRT (or simply FRT) is well studied in the literature [1,59-67]. Complex FRTs are FRTs whose order parameter is complex [34-38].

When the order is an imaginary number $i b$, then we obtain the following special case of CLCTs with the transform matrix:

$$
\mathbf{F}_{\mathrm{lc}}^{i b}=\left[\begin{array}{cc}
\cosh (b \pi / 2) & i \sinh (b \pi / 2) \\
-i \sinh (b \pi / 2) & \cosh (b \pi / 2)
\end{array}\right],
$$

which again differs only by the factor $e^{b \pi / 4}$ from FRTs as commonly defined,

$$
\mathcal{C}_{\mathbf{F}_{\mathrm{lc}}^{i b f}} f(u)=\mathcal{F}_{\mathrm{lc}}^{i b} f(u)=e^{b \pi / 4} \mathcal{F}^{i b} f(u) .
$$

Since FRTs are additive in index, a real-ordered and a purely imaginary-ordered FRT can be combined as $\mathcal{F}^{a+i b}$ $=\mathcal{F}^{a} \mathcal{F}^{i b}$ to yield a general CFRT, where the complex order may be denoted by $a_{c}=a+i b$. CFRTs can be optically realized by using thin lenses, free-space propagations, and Gaussian apertures or by combination of Gauss- 
Weierstrass transforms with Gaussian apertures [34].

\section{ALGORITHM}

We now show how given abcd matrices can be decomposed in a manner that leads to a fast algorithm for the computation of CLCTs. In the most general case, the matrix $\mathbf{M}_{C}$ is composed of the four complex parameters $a, b, c, d$, whose real and imaginary parts add up to a total of eight parameters. These eight parameters are restricted by the unimodularity condition on $\mathbf{M}_{C}$, which requires the real part of the determinant to be 1 and the imaginary part to be zero. Because of these two equations, the total number of independent parameters of a general CLCT is 6. These six parameters correspond to the six parameters of the group $H S p(2, \mathrm{C})$, which is a six-parameter semigroup of the complex symplectic group $S p(2, \mathrm{C})$. Before giving the main decomposition which covers the general case, we start with a special case whose treatment is straightforward.

\section{A. $b=0$ Case}

When $b=0$, the unimodularity requirement requires $a$ $\neq 0$ and the transform output can be written as

$$
\left(\mathcal{C}_{\mathbf{M}_{C}} f\right)(u)=\frac{1}{\sqrt{a}} e^{j c y^{2} / 2 a} f(y / a)
$$

In this case, the output is given by a scaling operation with parameter $a$ followed by a CM operation with parameter $-c / 2 a$. We will restrict ourselves to the case where $a$ is real since only in this case will the scaling operation result in a $R \rightarrow R$ mapping. The case where $a$ is complex produces complex scaling operations and therefore causes mappings from functions on the real line to functions on the complex plane. This case would require special treatment, which we do not attempt since we are not aware of any optical realization or application of such transforms. Also necessary is the condition $\operatorname{Im}(c / a)$, which is necessary to ensure boundedness. In order to have a bounded and $\mathbb{R} \rightarrow \mathbb{R}$ mapping, it becomes necessary for $a$ to be real and $\operatorname{Im}(c / a) \geq 0$. Together with the unitdeterminant condition, these conditions can be explicitly summarized as follows:

$$
\begin{gathered}
a_{r} \neq 0, \\
d=1 / a, \\
a_{c}=0, \\
a_{r} c_{c} \geq 0,
\end{gathered}
$$

where the first two are intrinsically required to define any LCT (det $\mathbf{M}_{C}=1$ ) and the last two are required to obtain a bounded $\mathbb{R} \rightarrow \mathbb{R}$ mapping. When the conditions in 23 are satisfied, the matrix $\mathbf{M}_{C}$ can be decomposed as

$$
\begin{aligned}
\mathbf{M}_{C}= & {\left[\begin{array}{cc}
a_{r} & 0 \\
c & 1 / a_{r}
\end{array}\right]=\left[\begin{array}{cc}
1 & 0 \\
c / a_{r} & 1
\end{array}\right]\left[\begin{array}{cc}
a_{r} & 0 \\
0 & 1 / a_{r}
\end{array}\right]=\left[\begin{array}{cc}
1 & 0 \\
c_{r} / a_{r} & 1
\end{array}\right] } \\
& \times\left[\begin{array}{cc}
1 & 0 \\
i c_{c} / a_{r} & 1
\end{array}\right]\left[\begin{array}{cc}
a_{r} & 0 \\
0 & 1 / a_{r}
\end{array}\right]
\end{aligned}
$$

The above decomposition can be used for the fast computation of the special case $b=0$.

\section{B. $b \neq 0$ Case}

Now, we turn our attention to the more general case in which the following decomposition will be the basis of our fast algorithm:

$$
\begin{aligned}
\mathbf{M}_{C}= & {\left[\begin{array}{cc}
1 & 0 \\
-q_{3 r} & 1
\end{array}\right]\left[\begin{array}{cc}
1 & 0 \\
-i q_{3 c} & 1
\end{array}\right]\left[\begin{array}{cc}
0 & -1 \\
1 & 0
\end{array}\right]\left[\begin{array}{cc}
1 & 0 \\
-q_{2 r} & 1
\end{array}\right]\left[\begin{array}{cc}
1 & 0 \\
-i q_{2 c} & 1
\end{array}\right] } \\
& \times\left[\begin{array}{cc}
0 & 1 \\
-1 & 0
\end{array}\right]\left[\begin{array}{cc}
1 & 0 \\
-q_{1 r} & 1
\end{array}\right]\left[\begin{array}{cc}
1 & 0 \\
-i q_{1 c} & 1
\end{array}\right] .
\end{aligned}
$$

This decomposition consists of three imaginary CM and real CM pairs with Fourier/inverse Fourier transform operations in between. The imaginary $\mathrm{CM}$ and real CM pairs can also be viewed as CCM operations,

$$
\begin{aligned}
\mathbf{M}_{C}= & {\left[\begin{array}{cc}
1 & 0 \\
-\left(q_{3 r}+i q_{3 c}\right) & 1
\end{array}\right]\left[\begin{array}{cc}
0 & -1 \\
1 & 0
\end{array}\right]\left[\begin{array}{cc}
1 & 0 \\
-\left(q_{2 r}+i q_{2 c}\right) & 1
\end{array}\right] } \\
& \times\left[\begin{array}{cc}
0 & 1 \\
-1 & 0
\end{array}\right]\left[\begin{array}{cc}
1 & 0 \\
-\left(q_{1 r}+i q_{1 c}\right) & 1
\end{array}\right]
\end{aligned}
$$

The three matrices in the center can also be expressed as a CCC operation,

$\mathbf{M}_{C}=\left[\begin{array}{cc}1 & 0 \\ -\left(q_{3 r}+i q_{3 c}\right) & 1\end{array}\right]\left[\begin{array}{cc}1 & \left(q_{2 r}+i q_{2 c}\right) \\ 0 & 1\end{array}\right]\left[\begin{array}{cc}1 & 0 \\ -\left(q_{1 r}+i q_{1 c}\right) & 1\end{array}\right]$,

which is nothing but the complex version of the wellknown CM-CC-CM decomposition [1].

When we multiply out the matrices on the right-hand side of Eq. (25), equate the result to the general CLCT matrix given in Eq. (6), and solve for our decomposition parameters in terms of the CLCT parameters, we get the following:

$$
\begin{array}{r}
q_{1 r}=\frac{b_{r}-b_{r} a_{r}-a_{c} b_{c}}{b_{r}^{2}+b_{c}^{2}}, \\
q_{1 c}=\frac{b_{c} a_{r}-b_{c}-b_{r} a_{c}}{b_{r}^{2}+b_{c}^{2}}, \\
q_{2 r}=b_{r}, \\
q_{2 c}=b_{c}, \\
q_{3 r}=\frac{b_{r}-b_{r} d_{r}-d_{c} b_{c}}{b_{r}^{2}+b_{c}^{2}},
\end{array}
$$




$$
q_{3 c}=\frac{b_{c} d_{r}-b_{c}-b_{r} d_{c}}{b_{r}^{2}+b_{c}^{2}} .
$$

Thus, all six parameters of our decomposition have been expressed in terms of the six parameters of the CLCT which we desire to compute. By using Eq. (9), we can also easily calculate the decomposition parameters in terms of the complex $\alpha, \beta, \gamma$ parameters if needed. The decomposition in Eq. (25) can also be expressed in operator notation as follows:

$$
\mathcal{C}_{\mathbf{M}}=\mathcal{Q}_{q_{3 r}} \mathcal{Q}_{i q_{3 c}} \mathcal{F}_{\mathrm{lc}}^{-1} \mathcal{Q}_{q_{2 r}} \mathcal{Q}_{i q_{2 c}} \mathcal{F}_{\mathrm{lc}} \mathcal{Q}_{q_{1 r}} \mathcal{Q}_{i q_{1 c}} .
$$

We now discuss the various cases that arise depending on the values of the parameters. When $b \neq 0$, a separate treatment is required depending on whether $a$ is zero or not. First, consider the case when $a=0$. The decomposition parameters given in Eq. (28) become

$$
\begin{gathered}
q_{1 r}=\frac{b_{r}}{b_{r}^{2}+b_{c}^{2}}, \\
q_{1 c}=\frac{-b_{c}}{b_{r}^{2}+b_{c}^{2}}, \\
q_{2 r}=b_{r}, \\
q_{3 r}=\frac{b_{2 c}=b_{c},}{b_{r}^{2}+b_{r}^{2} d_{r}-d_{c} b_{c}}, \\
q_{3 c}=\frac{b_{c} d_{r}-b_{c}-b_{r} d_{c}}{b_{r}^{2}+b_{c}^{2}} .
\end{gathered}
$$

As discussed in Subsection 3.B.2, the CCM parameters $q_{1 c}, q_{2 c}$, and $q_{3 c}$ should be $\leq 0$ leading to the conditions

$$
\begin{gathered}
\frac{-b_{c}}{b_{r}^{2}+b_{c}^{2}} \leq 0, \\
b_{c} \leq 0, \\
\frac{b_{c} d_{r}-b_{c}-b_{r} d_{c}}{b_{r}^{2}+b_{c}^{2}} \leq 0 .
\end{gathered}
$$

Equations (31) and (32) imply $b_{c}=0$ and Eq. (33) becomes $b_{r} d_{c} \geq 0$. When we set $b_{c}=0$ in Eq. (30), we obtain the following decomposition parameters:

$$
\begin{gathered}
q_{1 r}=1 / b_{r}, \\
q_{1 c}=0, \\
q_{2 r}=b_{r}, \\
q_{2 c}=0, \\
q_{3 r}=\left(1-d_{r}\right) / b_{r}^{2},
\end{gathered}
$$

$$
q_{3 c}=-d_{c} / b_{r},
$$

with the condition $b_{r} d_{c} \geq 0$. The decomposition we should use in this case therefore can be expressed as

$$
\begin{aligned}
\mathbf{M}_{C}= & {\left[\begin{array}{cc}
1 & 0 \\
-\left(1-d_{r}\right) / b_{r} & 1
\end{array}\right]\left[\begin{array}{cc}
1 & 0 \\
i d_{c} / b_{r} & 1
\end{array}\right]\left[\begin{array}{cc}
0 & -1 \\
1 & 0
\end{array}\right]\left[\begin{array}{cc}
1 & 0 \\
-b_{r} & 1
\end{array}\right] } \\
& \times\left[\begin{array}{cc}
0 & 1 \\
-1 & 0
\end{array}\right]\left[\begin{array}{cc}
1 & 0 \\
-1 / b_{r} & 1
\end{array}\right] .
\end{aligned}
$$

We now turn our attention to the case $b \neq 0$ and $a \neq 0$. The decomposition given in Eq. (25) and the decomposition parameters given in Eq. (28) are applicable. The below three conditions should be satisfied to have a bounded and $\mathrm{R} \rightarrow \mathbb{R}$ mapping:

$$
\begin{gathered}
b_{c} \leq 0, \\
b_{c} a_{r}-b_{r} a_{c} \leq b_{c}, \\
b_{c} d_{r}-b_{r} d_{c} \leq b_{c},
\end{gathered}
$$

which can be equivalently expressed in terms of the $\alpha, \beta, \gamma$ parameters,

$$
\begin{aligned}
& \beta_{c} \geq 0, \\
& \alpha_{c} \geq \beta_{c}, \\
& \gamma_{c} \geq \beta_{c},
\end{aligned}
$$

which depends only on the imaginary parts. This is expected since RLCTs are always bounded and unitary, and it is the imaginary parts that are involved in issues of boundedness. These conditions are derived by restricting the parameters of the Gaussian aperture steps in the CLCT decompositions we employ. There are no such conditions required for RLCTs. However, these constraints are crucial for the computation of CLCTs. To better illustrate these conditions, we summarize them in Table 1.

The special case $b=0$ requires the computation of only real $\mathrm{CM}$ and $\mathrm{CCM}$ and a real scaling operation. The decomposition for the general case includes CMs and FTs. $\mathrm{CM}$ s require only $N$ multiplications and can be done in $\sim N$ times. The FT and inverse FT can be computed in $\sim N \log N$ times by using the fast Fourier transform (FFT) algorithm. We also note that the scaling operation merely changes the sampling interval in the sense of reinterpretation of the same samples with a scaled sampling interval, in a manner which corresponds to scaling of the underlying continuous signal. Thus the cost of the scaling

Table 1. Summary of the Conditions to Have Bounded $\mathrm{R} \rightarrow \mathrm{R}$ CLCTs

\begin{tabular}{lcc}
\hline Case 1 & Case 2 & Case 3 \\
\hline$b=0$ & $b \neq 0$ and $a=0$ & $b \neq 0$ and $a \neq 0$ \\
$a_{r} \neq 0$ & $b_{c}=0$ & $b_{c} \leq 0$ \\
$d=1 / a$ & $b_{r} d_{c} \geq 0$ & $b_{c} a_{r}-b_{r} a_{c} \leq b_{c}$ \\
$a_{c}=0$ & & $b_{c} d_{r}-b_{r} d_{c} \leq b_{c}$ \\
$a_{r} c_{c} \geq 0$ & & \\
\hline
\end{tabular}




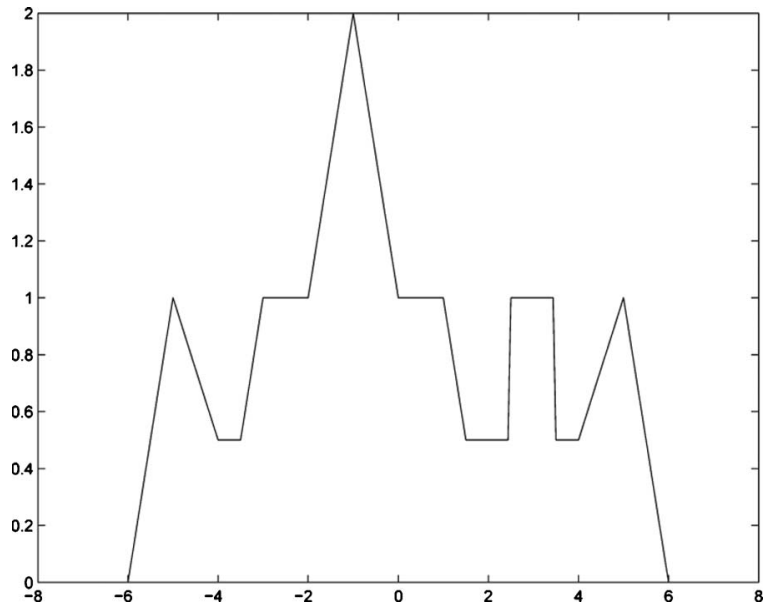

Fig. 2. Example function $F 4$.

operation is minimal and not of consequence since it amounts only to a reinterpretation of the samples. Therefore, the overall CLCT can be computed in $\sim N \log N$ times. To ensure that the number of samples required to represent the function is sufficient in the NyquistShannon sense at each step of the decomposition, we will track the space-bandwidth representation of the function by using the WD and increase the sampling rate when necessary. We will do this with the help of the procedures summarized in [47] for real steps and with the help of Fig. 1 and the discussion given in Subsection 3.B.2 for the complex components. Since the FRT corresponds to rotation and the scaling operation only to a reinterpretation of the samples, these steps never require us to increase the number of samples. CMs, however, require careful handling of the space-bandwidth and sampling issues.

Finally, we summarize our algorithm and the associated space-bandwidth product tracking and sampling control methodology for the most general case. [For the $b=0$ and $b \neq 0, a=0$ special cases, this procedure can be easily simplified to correspond to the simpler decompositions in Eqs. (24) and (35), respectively.] Whenever the current number of samples will not be sufficient to fully represent the operated-on signal in the Nyquist-Shannon sense, an increase in the number of samples is required prior to performing the operation.

1. We will use $E_{s}$ and $E_{f}$ to denote the spatial and frequency extent of the function as we go through the stages of the algorithm. We assume that the initial spacefrequency support is a square of edge length $\Delta u$ so that at the beginning $E_{s}=E_{f}=\Delta u$, and the signal can be represented with $E_{s} E_{f}=\Delta u^{2}$ samples.

2. The first step of the decomposition is the first CCM with parameter $q_{1 c}$. We use Eq. (16) to obtain the space and frequency extent of the Gaussian function, which we denote by $G_{s 1}$ and $G_{f 1}$, respectively. $E_{s}$ and $E_{f}$ are changed according to $E_{s} \rightarrow \min \left(E_{s}, G_{s 1}\right)$ and $E_{f} \rightarrow E_{s}+G_{f 1}$. The required number of samples then becomes $E_{s} \times E_{f}$ which are taken in the interval $\left[-\Delta E_{s} / 2, \Delta E_{s} / 2\right]$ with a spacing of $1 / E_{f}$ apart from each other. This may or may not require an increase in the number of samples depending on whether the new $E_{s} \times E_{f}$ product is bigger than the starting number of samples, $\Delta u^{2}$. If an increase in the number
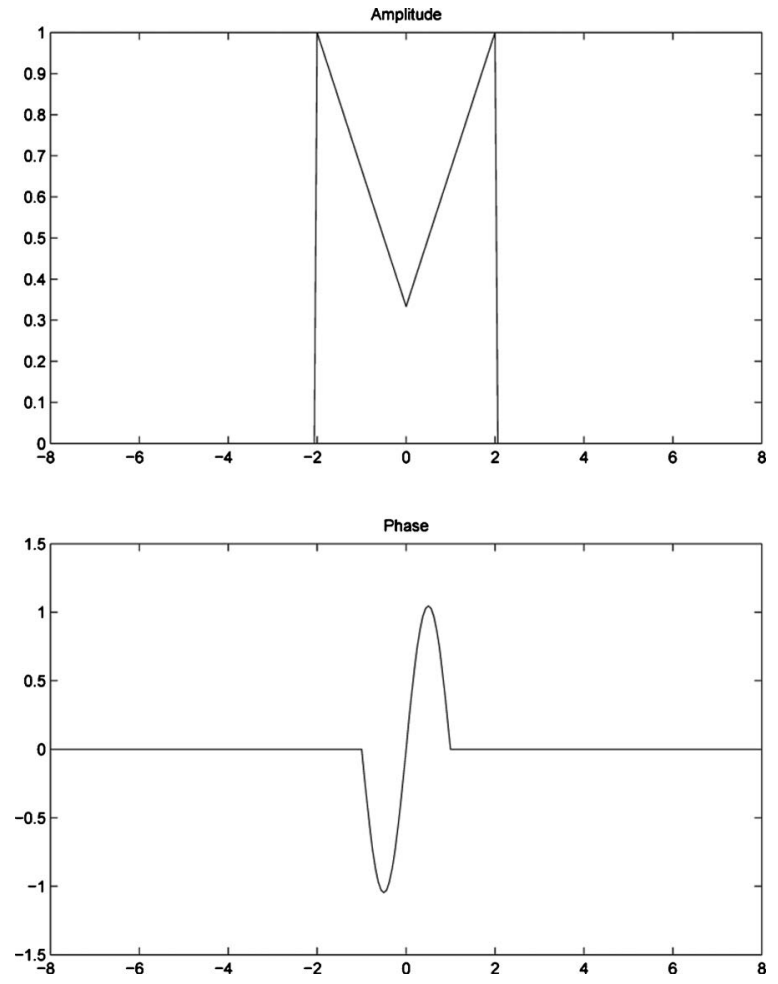

Fig. 3. Example function $F 5$.

of samples is required, we oversample the signal using an appropriate interpolation scheme and then the CCM operation is performed on the input samples.

3. The second step is a CM operation with parameter $q_{1 r}$. We see that the extent must now become $E_{s} \rightarrow E_{s}$ and $E_{f} \rightarrow E_{f}+\left|q_{1 r}\right| E_{s}$. The number of samples required becomes $E_{s} \times\left(E_{f}+\left|q_{1 r}\right| E_{s}\right)$ which will require oversampling with a factor $k=1+\left|q_{1 r}\right| E_{s} / E_{f}$. After this oversampling is performed, the CM operation is performed.

4. We now take the FT of the samples by using the FFT algorithm. We have $E_{s} \rightarrow E_{f}$ and $E_{f} \rightarrow E_{s}$ since FT only switches the spatial variable and its spatial-frequency variable. The FT operation does not change the spacebandwidth product of the signal, so oversampling is not required at this stage.

5. Repeat Steps 2 and 3 with the parameters $q_{2 c}$ and $q_{2 r}$ corresponding to subsequent stages of the decomposition.

6. Repeat Step 4, this time with an inverse FT operation instead of a forward FT operation.

7. Repeat Steps 2 and 3 with the parameters $q_{3 c}$ and $q_{3 r}$ corresponding to the final stages of the decomposition to get the final output samples.

\section{NUMERICAL EXAMPLES}

We have considered several examples to illustrate the performance of the presented algorithm. We consider the chirped pulse function $\exp \left(-\pi u^{2}-i \pi u^{2}\right)$, denoted by $F 1$, and the trapezoidal function $1.5 \operatorname{tri}(u / 3)-0.5 \operatorname{tri}(u)$, denoted by $F 2[\operatorname{tri}(u)=\operatorname{rect}(u) * \operatorname{rect}(u)]$. Since these two functions are well confined to a circle in the spacefrequency plane with a diameter of $\Delta u=8$, we take $N=8^{2}$. 
We also consider the binary sequence 01101010 occupying $[-8,8]$ with each bit 2 units in length so that $N=16^{2}$. This binary sequence is denoted by $F 3$ and the function shown in Fig. 2 is denoted by $F 4$, again with $N=16^{2}$. Additionally, we also test the example function given in Fig. 3 with $N=8^{2}$ that has complex values (i.e., amplitude and phase). These choices for $\Delta u$ result in $\sim 0 \%, 0.0002 \%$, $0.47 \%, 0.03 \%$, and $0.25 \%$ of the energies of $F 1, F 2, F 3, F 4$, and $F 5$, respectively, to fall outside the chosen frequency extent. The chosen space extent includes all of the energies of $F 2, F 3, F 4$, and $F 5$ and virtually all of the energy of $F 1$. We consider three transforms: the first $(T 1)$ with parameters $\left(\alpha_{r}, \beta_{r}, \gamma_{r} ; \alpha_{c}, \beta_{c}, \gamma_{c}\right)=(-2,1.2,-0.9 ; 0.04,0.02$, $0.12)$, the second (T2) with parameters $(1.15,-0.14$, $-0.1 ; 0.003,0.001,0.002)$, and the third (T3) with parameters $(-1.2,-0.3,0.1 ; 0.6,0.5,1)$. The CLCTs $T 1, T 2$, and $T 3$ of the functions $F 1, F 2, F 3, F 4$, and $F 5$ have been computed both by the presented fast algorithm and by a highly inefficient brute force numerical approach based on Simpson's numerical integration, which is taken here as a reference.

The results for all functions $(F 1, F 2, F 3, F 4, F 5)$ are plotted in Figs. 4 and 5 for transforms $T 1$ and $T 2$, respec- tively, and are tabulated in Table 2 for all transforms $T 1$, $T 2$, and T3. Also shown are the errors that arise when using the discrete Fourier transform (DFT) in approximating the FT of the same functions, which serves as a reference. (The error is defined as the energy of the difference normalized by the energy of the reference, expressed as a percentage.)

We also tested our algorithm for the CFRT, which is an important special case of CLCTs and the complex extension of the real-parametered FRT. A CFRT with order $0.8-i 0.2$ is calculated with our algorithm and the reference method and the results are plotted for all functions $(F 1, F 2, F 3, F 4, F 5)$ in Fig. 6 and again are tabulated in Table 2. The CFRT order $0.8-i 0.2$ corresponds to CLCT parameters $\quad\left(\alpha_{r}, \beta_{r}, \gamma_{r} ; \alpha_{c}, \beta_{c}, \gamma_{c}\right)=(0.292,0.9919,0.292$; 0.3331, 0.098, 0.3331).

Examination of the table shows that our algorithm can accurately compute CLCTs for a variety of transforms and functions. We observe that the main determinant of the error is not the transform, but the function, and more specifically the energy of the function lying outside the assumed extent. If we require the error to be further re-
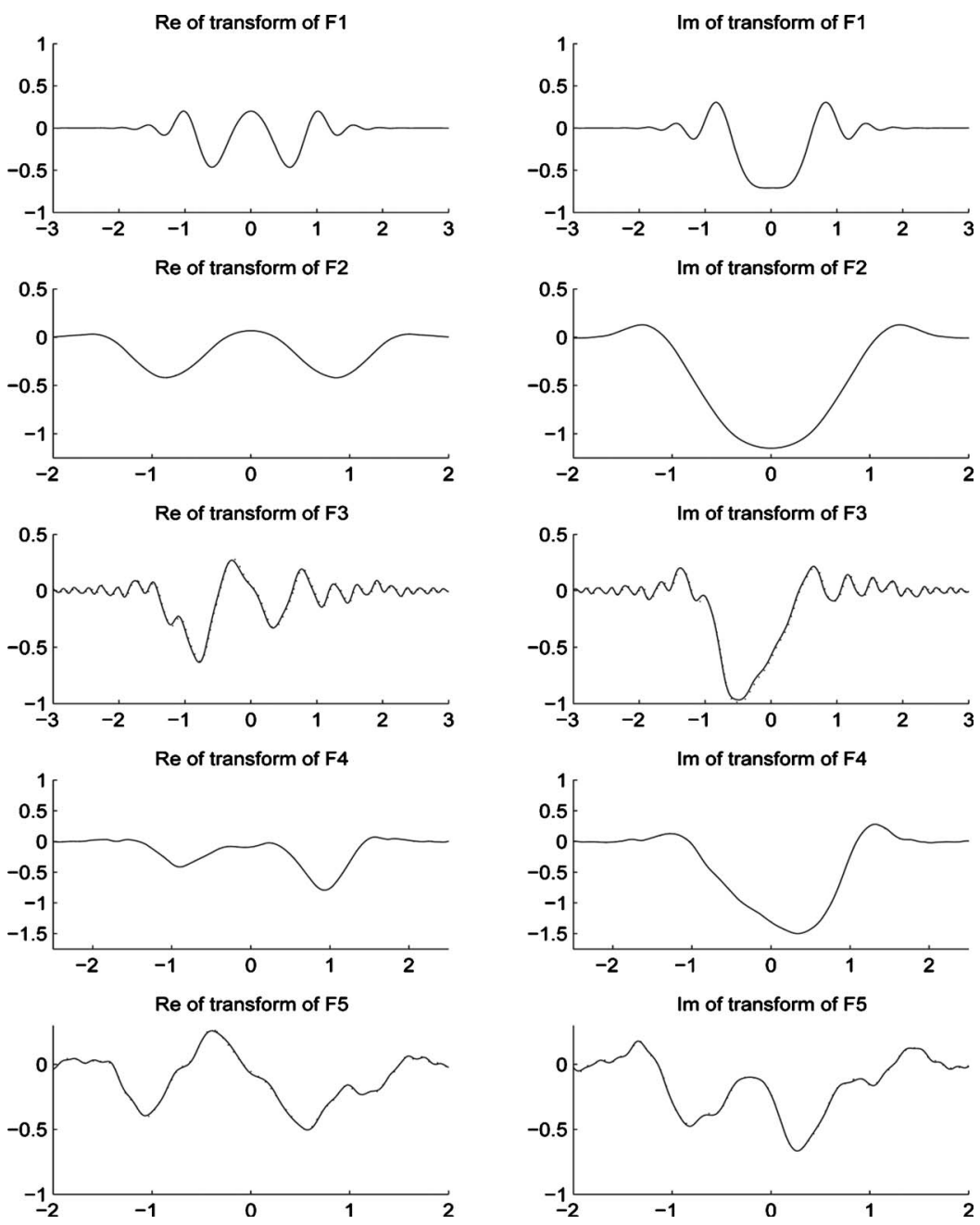

Fig. 4. Transform (T1) of $F 1, F 2, F 3, F 4$, and $F 5$. The results obtained with the presented algorithm and the reference result have been plotted with dotted and solid lines, respectively. However, the two types of lines are almost indistinguishable since the results are very close. 
Re of transform of F1
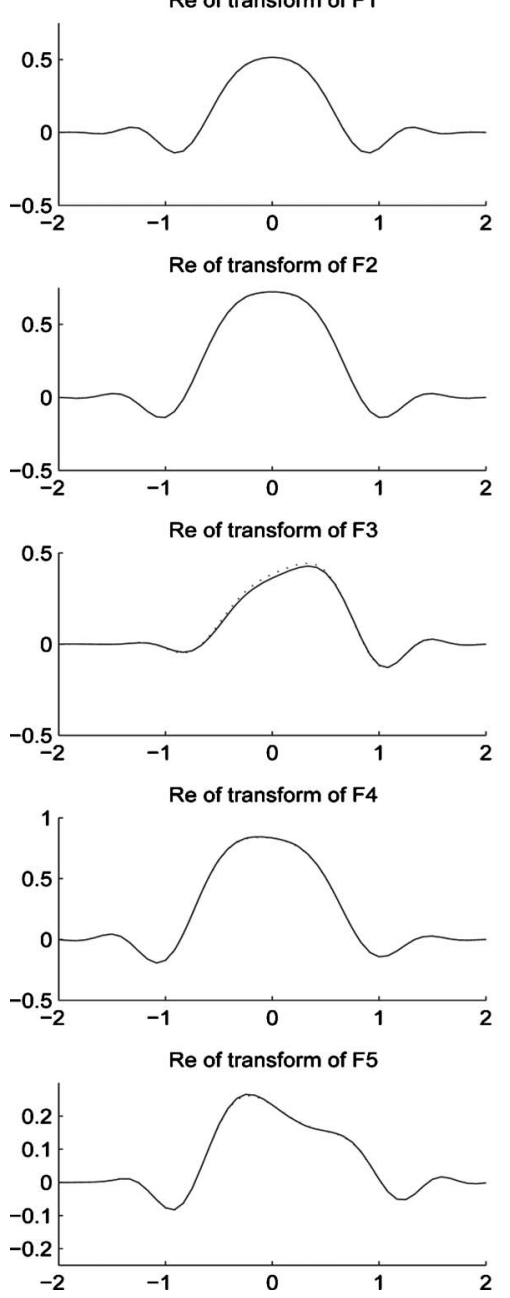

Im of transform of F1
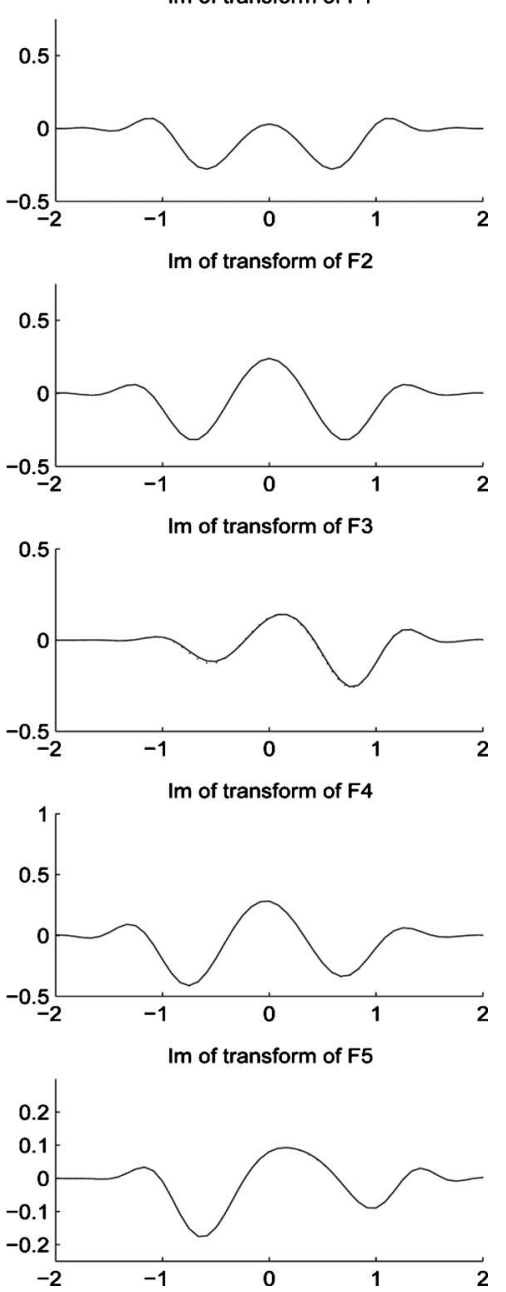

Fig. 5. Transform (T3) of $F 1, F 2, F 3, F 4$, and $F 5$. The results obtained with the presented algorithm and the reference result have been plotted with dotted and solid lines, respectively. However, the two types of lines are almost indistinguishable since the results are very close.

duced, we can reduce the excluded energy by increasing the extent and the number of samples involved.

\section{CONCLUSIONS}

We presented an algorithm for the fast and accurate digital computation of the general family of complexparametered linear canonical transforms (LCTs). This family of transform integrals can represent a general class of complex quadratic-phase systems in optics. Our approach is based on concepts from signal analysis and processing rather than conventional numerical analysis. With careful consideration of sampling issues, the num- ber of samples, $N$, can be chosen very close to the spacebandwidth product of the functions. A naive approach based on the examination of the frequency content of the integral kernels would, on the other hand, result in an unnecessarily high number of samples being taken due to the highly oscillatory nature of the kernels, which would not only be representationally inefficient but also increase the computation time and storage requirements. The transform output may have a higher space-bandwidth product than the input due to the nature of the transform family. Through careful space-bandwidth tracking and control, we can assure that the output samples obtained are accurate approximations to the true ones and that

Table 2. Percentage Errors for Different Functions $\boldsymbol{F}$ and Transforms $T$

\begin{tabular}{lccccc}
\hline & $T 1$ & $T 2$ & $T 3$ & CFRT & DFT \\
\hline$F 1$ & $4.12 \times 10^{-6}$ & $2.19 \times 10^{-6}$ & $2.8 \times 10^{-3}$ & $1.24 \times 10^{-5}$ & $2.0 \times 10^{-21}$ \\
$F 2$ & $3.73 \times 10^{-4}$ & $7.1 \times 10^{-3}$ & $1.4 \times 10^{-3}$ & $1.2 \times 10^{-3}$ & $6.2 \times 10^{-4}$ \\
$F 3$ & 0.53 & 0.35 & 0.26 & 0.22 & 1.2 \\
$F 4$ & $1.2 \times 10^{-3}$ & $4.96 \times 10^{-2}$ & $2.0 \times 10^{-3}$ & $2.2 \times 10^{-3}$ & $7.1 \times 10^{-2}$ \\
$F 5$ & 0.11 & 0.2 & $8.0 \times 10^{-3}$ & $6.4 \times 10^{-3}$ & 1.7 \\
\hline
\end{tabular}


$\operatorname{Re}$ of CFRT of F1
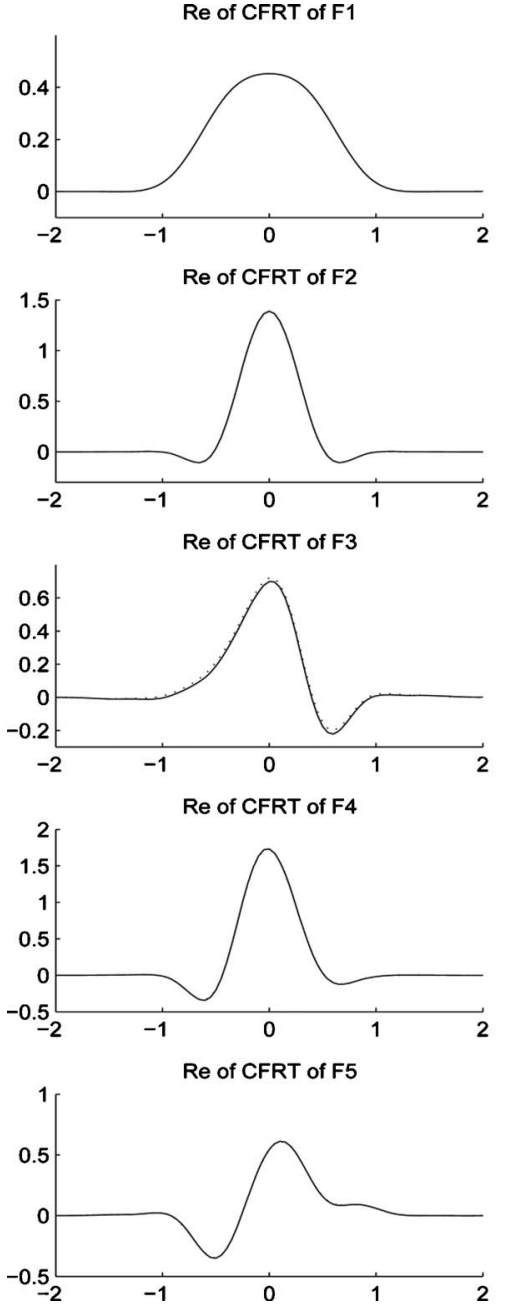
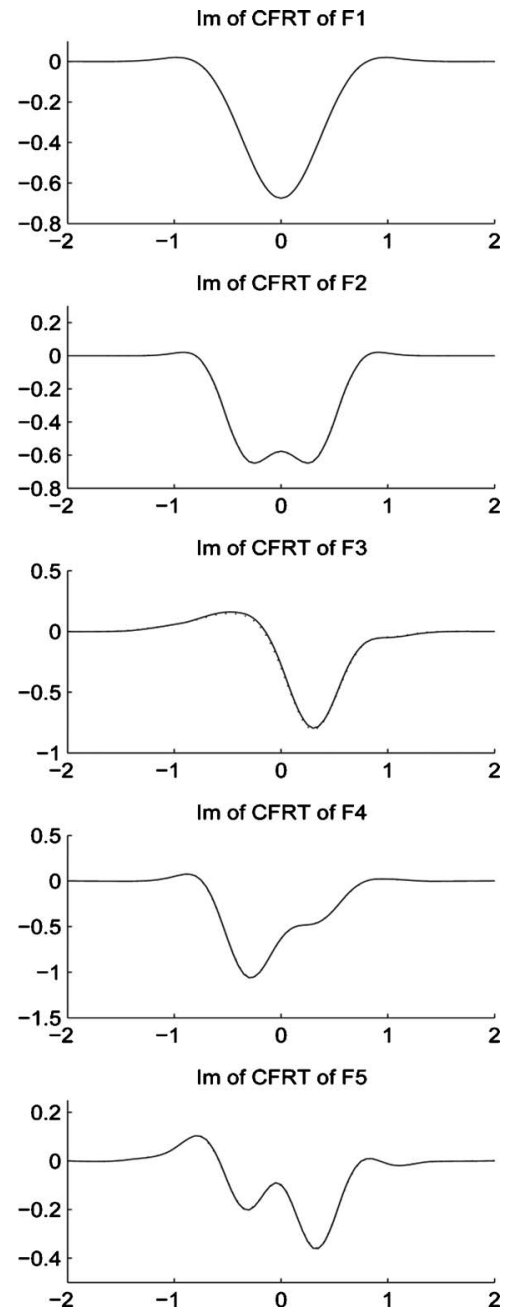

Fig. 6. CFRT with order $0.8-i 0.2$ of $F 1, F 2, F 3, F 4$, and $F 5$. The results obtained with the presented algorithm and the reference result have been plotted with dotted and solid lines, respectively. However, the two types of lines are almost indistinguishable since the results are very close.

they are sufficient (but not unnecessarily redundant) in the Nyquist-Shannon sense, allowing a full reconstruction of the underlying continuous output functions. The algorithm takes the samples of the input function and maps them to the samples of the continuous CLCT of this function in the same sense that the fast Fourier transform (FFT) implementation of the DFT computes the samples of the continuous FT of a function.

Complex-parametered LCTs allow several kinds of optical systems to be represented, including lossy as well as lossless ones. When complex parameters are involved, LCTs may no longer be unitary and boundedness issues may arise. We have identified the conditions for a CLCT to constitute a bounded map from functions on the real axis to functions on the real axis. As a special case of our general CLCT algorithm, we have also obtained an efficient and accurate algorithm for complex-ordered fractional Fourier transforms (CFRTs).

\section{ACKNOWLEDGMENTS}

A. Koç and L. Hesselink gratefully acknowledge support from the Research Laboratories at the General Electric
(GE) Corporation in New York. H. M. Ozaktas acknowledges partial support of the Turkish Academy of Sciences.

Note added on proof: We have recently been made aware of a new work [68] on the subject of fast LCT computation.

\section{REFERENCES}

1. H. M. Ozaktas, Z. Zalevsky, and M. A. Kutay, The Fractional Fourier Transform with Applications in Optics and Signal Processing (Wiley, 2001).

2. A. E. Siegman, Lasers (University Science Books, 1986).

3. M. J. Bastiaans, "The Wigner distribution function applied to optical signals and systems," Opt. Commun. 25, 26-30 (1978).

4. K. B. Wolf, "Construction and properties of canonical transforms," in Integral Transforms in Science and Engineering (Plenum, 1979), Chap. 9 .

5. M. Moshinsky, "Canonical transformations and quantum mechanics," SIAM J. Appl. Math. 25, 193-212 (1973).

6. C. Jung and H. Kruger, "Representation of quantum mechanical wavefunctions by complex valued extensions of classical canonical transformation generators," J. Phys. A 15, 3509-3523 (1982).

7. B. Davies, Integral Transforms and Their Applications (Springer, 1978). 
8. D. J. Griffiths and C. A. Steinke, "Waves in locally periodic media," Am. J. Phys. 69, 137-154 (2001).

9. D. W. L. Sprung, H. Wu, and J. Martorell, "Scattering by a finite periodic potential," Am. J. Phys. 61, 1118-1124 (1993).

10. L. L. Sanchez-Soto, J. F. Carinena, A. G. Barriuso, and J. J. Monzon, "Vector-like representation of one-dimensional scattering," Eur. J. Phys. 26, 469-480 (2005).

11. S. Baskal and Y. S. Kim, "Lens optics as an optical computer for group contractions," Phys. Rev. E 67, 056601 (2003).

12. S. Baskal and Y. S. Kim, "ABCD matrices as similarity transformations of Wigner matrices and periodic systems in optics," J. Opt. Soc. Am. A 26, 2049-2054 (2009).

13. E. Georgieva and Y. S. Kim, "Slide-rule-like property of Wigner's little groups and cyclic $\mathrm{S}$ matrices for multilayer optics," Phys. Rev. E 68, 026606 (2003).

14. M. Moshinsky and C. Quesne, "Linear canonical transformations and their unitary representations," J. Math. Phys. 12, 1772-1780 (1971)

15. M. J. Bastiaans, "Wigner distribution function and its application to first-order optics," J. Opt. Soc. Am. 69, 17101716 (1979).

16. M. J. Bastiaans, Applications of the Wigner Distribution Function in Optics, The Wigner Distribution: Theory and Applications in Signal Processing (Elsevier, 1997), pp. 375426.

17. S. Abe and J. T. Sheridan, "Generalization of the fractional Fourier transformation to an arbitrary linear lossless transformation an operator approach,” J. Phys. A 27, 4179_ 4187 (1994).

18. S. Abe and J. T. Sheridan, "Optical operations on wavefunctions as the Abelian subgroups of the special affine Fourier transformation," Opt. Lett. 19, 1801-1803 (1994).

19. M. J. Bastiaans and T. Alieva, "Classification of lossless first-order optical systems and the linear canonical transformation," J. Opt. Soc. Am. A 24, 1053-1062 (2007).

20. M. J. Bastiaans and T. Alieva, "Synthesis of an arbitrary ABCD system with fixed lens positions," Opt. Lett. 31, 2414-2416 (2006).

21. A. Sahin, H. M. Ozaktas, and D. Mendlovic, "Optical implementations of two-dimensional fractional Fourier transforms and linear canonical transforms with arbitrary parameters,” Appl. Opt. 37, 2130-2141 (1998).

22. T. Alieva and M. J. Bastiaans, "Properties of the canonical integral transformation,” J. Opt. Soc. Am. A 24, 3658-3665 (2007)

23. J. Rodrigo, T. Alieva, and M. L. Calvo, "Optical system design for orthosymplectic transformations in phase space," $\mathrm{J}$ Opt. Soc. Am. A 23, 2494-2500 (2006).

24. J. A. Rodrigo, T. Alieva, and M. L. Calvo, "Gyrator transform: properties and applications," Opt. Express 15, 21902203 (2007)

25. J. A. Rodrigo, T. Alieva, and M. L. Calvo, "Experimental implementation of the gyrator transform," J. Opt. Soc. Am. A 24, 3135-3139 (2007)

26. U. Sümbül and H. M. Ozaktas, "Fractional free space, fractional lenses, and fractional imaging systems," J. Opt. Soc. Am. A 20, 2033-2040 (2003).

27. K. B. Wolf, "Canonical transformations I. Complex linear transforms," J. Math. Phys. 15, 1295-1301 (1974).

28. K. B. Wolf, "Canonical transformations II. Complex radial transforms,” J. Math. Phys. 15, 2102-2111 (1974).

29. P. Kramer, M. Moshinsky, and T. H. Seligman, Complex Extensions of Canonical Transformations and Quantum Mechanics, Vol. 3 of Group Theory and Its Applications, E. M. Loebl, ed. (Academic, 1975), pp. 249-332.

30. V. Bargmann, "On a Hilbert space of analytic functions and an associated integral transform, part I," Commun. Pure Appl. Math. 14, 187-214 (1961).

31. V. Bargmann, "On a Hilbert space of analytic functions and an associated integral transform, part II," Commun. Pure Appl. Math. 20, 1-101 (1967).

32. K. B. Wolf, "On self-reciprocal functions under a class of integral transforms," J. Math. Phys. 18, 1046-1051 (1977).
33. A. Torre, "Linear and radial canonical transforms of fractional order," J. Comput. Appl. Math. 153, 477-486 (2003).

34. C.-C. Shih, "Optical interpretation of a complex-order Fourier transform," Opt. Lett. 20, 1178-1180 (1995).

35. L. M. Bernardo and O. D. D. Soares, "Optical fractional Fourier transforms with complex orders," Appl. Opt. 35, 3163-3166 (1996).

36. C. Wang and B. Lü, "Implementation of complex-order Fourier transforms in complex ABCD optical systems," Opt. Commun. 203, 61-66 (2002).

37. L. M. Bernardo, "Talbot self-imaging in fractional Fourier planes of real and complex orders," Opt. Commun. 140, 195-198 (1997).

38. A. A. Malyutin, "Complex-order fractional Fourier transforms in optical schemes with Gaussian apertures," Quantum Electron. 34, 960-964 (2004).

39. L. M. Bernardo, "ABCD matrix formalism of fractional Fourier optics,” Opt. Eng. (Bellingham) 35, 732-740 (1996).

40. H. Fan, L. Hu, and J. Wang, "Eigenfunctions of the complex fractional Fourier transform obtained in the context of quantum optics," J. Opt. Soc. Am. A 25, 974-978 (2008).

41. D. Dragoman, "Classical versus complex fractional Fourier transformation," J. Opt. Soc. Am. A 26, 274-277 (2009).

42. A. Koç, H. M. Ozaktas, and L. Hesselink, "Fast and accurate computation of two-dimensional non-separable quadratic-phase integrals," J. Opt. Soc. Am. A 27, 12881302 (2010).

43. B. M. Hennelly and J. T. Sheridan, "Fast numerical algorithm for the linear canonical transform," J. Opt. Soc. Am. A 22, 928-937 (2005).

44. B. M. Hennelly and J. T. Sheridan, "Generalizing, optimizing, and inventing numerical algorithms for the fractional Fourier, Fresnel, and linear canonical transforms," J. Opt. Soc. Am. A 22, 917-927 (2005).

45. H. M. Ozaktas, O. Arıkan, M. A. Kutay, and G. Bozdağı, "Digital computation of the fractional Fourier transform," IEEE Trans. Signal Process. 44, 2141-2150 (1996).

46. H. M. Ozaktas, A. Koç, I. Sari, and M. A. Kutay, "Efficient computation of quadratic-phase integrals in optics," Opt. Lett. 31, 35-37 (2006).

47. A. Koç, H. M. Ozaktas, C. Candan, and M. A. Kutay, "Digital computation of linear canonical transforms," IEEE Trans. Signal Process. 56, 2383-2394 (2008).

48. F. S. Oktem and H. M. Ozaktas, "Exact relation between continuous and discrete linear canonical transforms," IEEE Signal Process. Lett. 16, 727-730 (2009).

49. J. J. Healy and J. T. Sheridan, "Cases where the linear canonical transform of a signal has compact support or is band-limited," Opt. Lett. 33, 228-230 (2008).

50. J. J. Healy, B. M. Hennelly, and J. T. Sheridan, "Additional sampling criterion for the linear canonical transform," Opt. Lett. 33, 2599-2601 (2008).

51. J. J. Healy and J. T. Sheridan, "Sampling and discretization of the linear canonical transform," Signal Process. 89, 641648 (2009).

52. J. Healy and J. T. Sheridan, "Fast linear canonical transforms," J. Opt. Soc. Am. A 27, 21-30 (2010).

53. F. Hlawatsch and G. F. Boudreaux-Bartels, "Linear and quadratic time-frequency signal representations," IEEE Signal Process. Mag. 9, 21-67 (1992).

54. L. Cohen, Time-Frequency Analysis (Prentice Hall, 1995)

55. A. W. Lohmann, R. G. Dorsch, D. Mendlovic, Z. Zalevsky, and C. Ferreira, "Space-bandwidth product of optical signals and systems," J. Opt. Soc. Am. A 13, 470-473 (1996).

56. B. Simon, "Resonances and complex scaling: A rigorous overview," Int. J. Quantum Chem. 14, 529-542 (1978).

57. J. N. Bardsley, "Complex scaling: An introduction," Int. J. Quantum Chem. 14, 343-352 (1978).

58. L. Onural, M. F. Erden, and H. M. Ozaktas, "Extensions to common Laplace and Fourier transforms," IEEE Signal Process. Lett. 4, 310-312 (1997).

59. D. Mendlovic and H. M. Ozaktas, "Fractional Fourier transforms and their optical implementation: I," J. Opt. Soc. Am. A 10, 1875-1881 (1993). 
60. H. M. Ozaktas and D. Mendlovic, "Fractional Fourier transforms and their optical implementation: II," J. Opt. Soc. Am. A 10, 2522-2531 (1993).

61. H. M. Ozaktas and D. Mendlovic, "Fourier transforms of fractional order and their optical interpretation," Opt. Commun. 101, 163-169 (1993).

62. H. M. Ozaktas, B. Barshan, D. Mendlovic, and L. Onural, "Convolution, filtering, and multiplexing in fractional Fourier domains and their relation to chirp and wavelet transforms," J. Opt. Soc. Am. A 11, 547-559 (1994).

63. L. B. Almeida, "The fractional Fourier transform and timefrequency representations," IEEE Trans. Signal Process. 42, 3084-3091 (1994).

64. H. M. Ozaktas and D. Mendlovic, "Fractional Fourier optics,” J. Opt. Soc. Am. A Opt. 12, 743-751 (1995).
65. H. M. Ozaktas and M. F. Erden, "Relationships among ray optical, Gaussian beam, and fractional Fourier transform descriptions of first-order optical systems," Opt. Commun. 143, 75-86 (1997).

66. A. Sahin, M. A. Kutay, and H. M. Ozaktas, "Nonseparable two-dimensional fractional Fourier transform," Appl. Opt. 37, 5444-5453 (1998).

67. A. Sahin, H. M. Ozaktas, and D. Mendlovic, "Optical implementation of the two-dimensional fractional Fourier transform with different orders in the two dimensions," Opt. Commun. 120, 134-138 (1995).

68. J. Healy and J. Sheridan, "Reevaluation of the direct method of calculating Fresnel and other linear canonical transforms," Opt. Lett. 35, 947-949 (2010). 